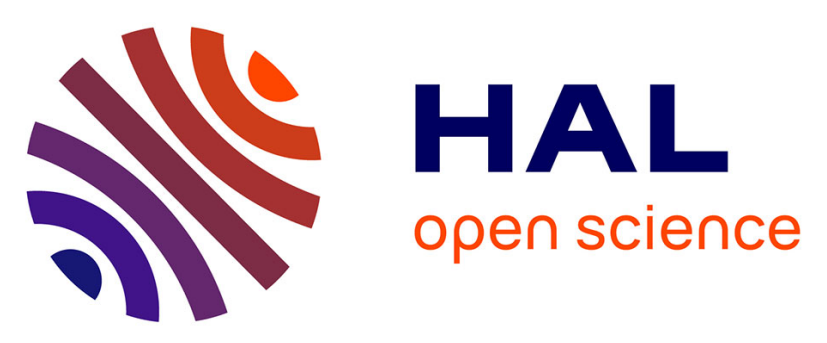

\title{
EyeSchool: an educational assistive technology for people with disabilities - Passing from single actors to multiple-actor environment
}

Cristina Popescu, Nadine Vigouroux, Mathieu Muratet, Julie Guillot, Petra Vlad, Frédéric Vella, Jawad Hajjam, Sylvie Ervé, Nathalie Louis, Julie Brin, et al.

\section{To cite this version:}

Cristina Popescu, Nadine Vigouroux, Mathieu Muratet, Julie Guillot, Petra Vlad, et al.. EyeSchool: an educational assistive technology for people with disabilities - Passing from single actors to multipleactor environment. 14th International Conference on Computers Helping People with Special Needs (ICCHP 2014), Jul 2014, Paris, France. pp.210-217, 10.1007/978-3-319-08599-9_32 . hal-01145825

\author{
HAL Id: hal-01145825 \\ https://hal.science/hal-01145825
}

Submitted on 27 Apr 2015

HAL is a multi-disciplinary open access archive for the deposit and dissemination of scientific research documents, whether they are published or not. The documents may come from teaching and research institutions in France or abroad, or from public or private research centers.
L'archive ouverte pluridisciplinaire HAL, est destinée au dépôt et à la diffusion de documents scientifiques de niveau recherche, publiés ou non, émanant des établissements d'enseignement et de recherche français ou étrangers, des laboratoires publics ou privés. 


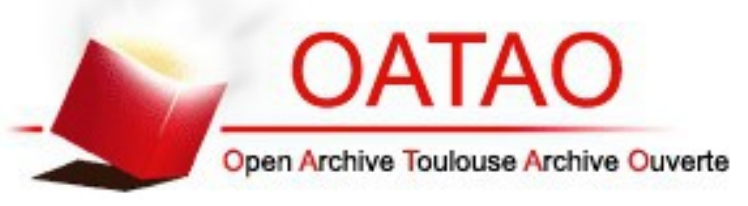

\section{Open Archive TOULOUSE Archive Ouverte (OATAO)}

OATAO is an open access repository that collects the work of Toulouse researchers and makes it freely available over the web where possible.

This is an author-deposited version published in : http://oatao.univ-toulouse.fr/ Eprints ID : 12999

To link to this article : DOI :10.1007/978-3-319-08599-9_32

URL : http://dx.doi.org/10.1007/978-3-319-08599-9 32

To cite this version : Popescu, Cristina and Vigouroux, Nadine and Muratet, Mathieu and Guillot, Julie and Vlad, Petra and Vella, Frédéric and Hajjam, Jawad and Ervé, Sylvie and Louis, Nathalie and Brin, Julie and Colineau, Joseph and Hobé, Thierry and Brimant, Loïc EyeSchool: an educational assistive technology for people with disabilities Passing from single actors to multiple-actor environment. (2014) In: International Conference on Computers Helping People with Special Needs - ICCHP 2014, 9 July 2014 - 11 July 2014 (Paris, France).

Any correspondance concerning this service should be sent to the repository administrator: staff-oatao@,listes-diff.inp-toulouse.fr 


\title{
EyeSchool: An Educational Assistive Technology for People with Disabilities - Passing from Single Actors to Multiple-Actor Environment
}

\author{
Cristina Popescu ${ }^{1}$, Nadine Vigouroux ${ }^{2}$, Mathieu Muratet ${ }^{1}$, Julie Guillot ${ }^{1}$, \\ Petra Vlad ${ }^{1}$, Frédéric Vella ${ }^{2}$, Jawad Hajjam ${ }^{3}$, Sylvie Ervé ${ }^{3}$, Nathalie Louis ${ }^{3}$, \\ Julie Brin ${ }^{3}$, Joseph Colineau ${ }^{4}$, Thierry Hobé ${ }^{5}$, and Loïc Brimant ${ }^{5}$ \\ 1 Grhapes (EA 7287) - INS HEA, 58-60 Avenue des Landes, 92150 Suresnes, France \\ 2 IRIT - Université Paul Sabatier, 118 route de Narbonne, \\ 31062 Toulouse Cedex 9, France \\ 3 CENTICH, 51 rue du Vallon, 49000 Angers, France \\ 4 Thales Research and Technology, Campus Polytechnique, 1 Avenue Augustin \\ Fresnel, 91767 Palaiseau Cedex, France \\ 5 Synerlog, 9 rue René Hersen, 49240 Avrille, France
}

\begin{abstract}
Since 2005, public policy in France has strongly been encouraging young people with disabilities inclusion within the regular school system. This has found a direct application through technical innovation, intended to help students being more independent within their learning activities. In this context, the purpose of this paper is to underline the manner in which using assistive information and communication technologies may improve the inclusive education for people with disabilities. The case study we present underlines the complexity of the social world into which the use of a precise assistive tool takes it place.
\end{abstract}

Keywords: Educational Assistive Technology, Notes-taking Tool, Inclusion, Multiple-actor Environment.

\section{Introduction}

Since 2005, public policy in France has strongly been encouraging young people with disabilities inclusion within the regular school system. This has addressed some new challenges for the "field" actors, as well as transformation within teaching practices. A human support is often needed in order to assist the student. In the same time, the different actors raised questions about the manner in which the educational content management should be improved. A direct application was then found through technical innovation, intended to help students being more independent within their learning activities.

Nonetheless, the paradigm of an average student as the final user of assistive technologies could hardly apply in this case, as students with disabilities have different kind of needs from the non-disabled but also between themselves. Specialised computer programs were for instance developed in order to be used by 
specific categories of people: for people with visual impairments: Zoomtext, Dolphin, Speakback, Wordread, Zoom-Ex, etc.; and for people with learning difficulties ScreenRuler, Medialexie, Speakback, Wordread, Cordial, Antidote, Skippy, etc.

Literature about the manner in which students with disabilities, and in general, people with disabilities are using assistive or "adapted" new technologies also tends to cluster around specific impairments, as visual [1],[4], hearing [7], physical or cognitive ones [3]. We use the term "adapted technologies" in order to designate the mainstream technologies whose uses were adapted to the needs of people with disabilities, for instance the general environment of Windows requiring special settings for people with visual impairments, etc.

Moreover, technologies and more precisely computer programs designed for education could be divided into the pedagogical ones - in general, teachers design exercises for their students, for instance "Langagiciel", "Genex" software, etc. and those encouraging the autonomy of their users - the students choose how to use and organise their contents, for instance the orDYScan ou AUSY projects.

In this context, the purpose of this presentation is to underline the manner in which using assistive information and communication technologies may improve the inclusive education for people with disabilities. We will therefore present a recently device developed for students with visual and hearing impairments and also with dyslexia, dyspraxia and dysphasia. It is also intended to encourage the autonomy of its users. The case study we present below underlines the complexity of the social world into which the use of this precise assistive tool takes it place.

\section{Description of EyeSchool Project}

The Eyeschool ${ }^{1}$ projet is supported by the French Department of Education and is funded by "Fonds national pour la société numérique". This project started in December 2012 with a two years duration. It includes four partners: CEN$\mathrm{TICH}$, a specialised centre for technologies for ageing people and for people with disabilities; Thales Group, a major electronic systems company; INS HEA, a specialised institute for the education of people with disabilities and Synerlog, a small company offering digital services.

As mentioned before, the project aims to test a digital notes-taking tool within a large population. It therefore means to encourage the participation of students with disabilities within their education environment. In this way, they may become more autonomous actors within their own notes-writing actions. Four French regions participate to the project. For each of them, one hundred students are expected to test the device during a school year period.

EyeSchool device is intended for three kinds of impairments at once: visual and hearing impairments and "dys" (dyslexia, developmental dyspraxia and dysphasia). All levels of education are covered: primary and secondary school as well as higher education. And these criteria may determine a universal device for people

${ }^{1}$ http://www.eyeschool.fr/ accessed April 3, 2014. 
with different kind of needs, but this will also bring challenges into its making and testing process.

\section{$3 \quad$ The Integrated-Package Description}

The EyeSchool package consists of hardware and software components. On the one hand, the hardware part counts on a computer, a webcam and a scanner. This is the main configuration as intended for a large number of users. But there are some other possible combinations like: a computer and an interactive whiteboard or a computer and a MimioCapture device.

On the other hand, the software solution offers multiple functionalities. The final user has the liberty to use them all or online a selection of them. An advanced setting system allows keeping inside the main interface only those functionalities one may consider interesting for him or her personal work.

Therefore, the EyeSchool main functionalities are the following ones: customising user interface: font size, colours, zoom, text-to-speech voice (used by people with visual impairments, dyslexia, dyspraxia and dysphasia) etc.; saving and modifying the blackboard image: contrast and luminosity level, zoom, colours filter, predefined colour options, etc.; scanning documents as image or as text with the help of an OCR software; editing and annotating text documents; adding notes to previously-saved images; help in organising contents (images, text documents and notes).

Even if for an experimental reason a specific webcam and portable scanner models were chosen, the software component is generally compatible with all TWAIN devices. SAPI compatibility is also available. Moreover, any video source recognised by Windows also works with EyeSchool image capturing software (Portanum).

In its present configuration, EyeSchool is first of all a mobile tool, less heavy and from an initial point of view, easier to be used or modified if one may compare it to other similar solutions. The fact of including different software tools (OCR, capturing and modifying images) in one single interface may bring a significant gaining in time. The note-taking system allows an easier classification of the documents.

EyeSchool was developed in order to respond to a mobility need, a relatively light system, easy to take into the classroom and that does not occupy too much place inside the school bag or on the table during school classes. The system was also initially designed in order to take less space on computers memory, as well as to have a low cost.

EyeSchool was build on the basis of an older free software for people with partial visual impairments, Portanum. This computer program allows saving images taken by a camera, modifying and adapting them to ones specific needs. There was nonetheless a less developed function from the notes taking point of view. EyeSchool were then designed in order to simplify this process. The scanner and OCR solutions appeared too. So from software initially intended for people with partial visual impairments, it could become a possible solution for other 
kind of specific needs. The fact of capturing the image of a blackboard seemed to be useful for people with dyslexia, especially when there were complex formulas or long texts. Having the possibility to quickly register this kind of contents might be for instance an opportunity for people with hearing impairments to concentrate on what it is transmitted by a sign language interpreter.

In the end, these entire hypotheses needed to be tested in a real situation. A large experimentation was designed and applied in four French regions.

\section{Description of the Initial Protocol}

An initial step-by-step action description of experimental actions was initially included within the project. The four participant regions were thus expected to follow it alike. To sum up, the protocol included three main phases. The first one was intended to last three months. It concerned the recruitment of students and their pedagogical and computer science referees. Their identification was imagined in a double way: through social health-care centres and public national education institutions, as well as Universities. Disability-specialised officials were expected to define the list of students and to inform the participant institutions.

Afterwards, a second phase as designed by the project was about organising regional meetings in order to present the project and train referees to install and to use EyeSchool device. These were considered as one-time events, lasting no longer than a half day.

Lastly, the third phase scheduled a one-time information meeting with public national educational institutions. The disability-specialised officials were expected to organise these meetings at the regional level.

Even if this protocol took into account some broad categories of actors, it tended to minimalize their diversity. Later on, we could see how same category actors behaved differently even if they were initially considered as similar. In the same time, some other participants like the students were not clearly defined as deciding actors within their recruitment.

We have chosen to detail the components of this step-by-step initial procedure in order to compare them to how testing was effectively done. One may consider this as unimportant in relationship with a computer program test, but we can discover that modification in actions determine for instance transformations in duration of use and therefore on the responses a participant might give about his of her use of the technical solution. Or how agenda unavailability might decide on the manner in which some people integrate or not the testing process.

\section{A Need to Observe the "Social World"}

Studies about assistive technologies for people with disabilities are mainly oriented towards technology assessment and/or laboratory trials in order to measure their utility and usability. They take into account the final users, the people with disabilities, but they give small details on the manner in which these individuals were found and how the people surrounding them may participate to 
the relationship they build with the assistive technologies. There are only few studies that focus on the manner in which these technologies are used in a "social" environment. And this could be mainly due to the complexity of the social world. If we take into account the actors involved in the EyeSchool deployment, we will see how they have different forms of expertise. Unexpected interactions and configurations can therefore occur between these same actors who do not hesitate to build their own "world". Especially when studying the use of a new assistive technology appears inside a network of some already existing relationships between school, child-care and family actors. And we will also see that this kind of elements cannot be anticipated or formalised in advance. Defining the studys fieldwork will thus become a complex activity.

Three different conceptions were therefore identified within our study of the impact of technology on education for people with disabilities: technology assessment, laboratory trials and social observations. Firstly, the technology assessment concerns the reliability of the device and of the software. Secondly, the laboratory observations are based on the interaction between the students and the object. And thirdly, the social observations add the family, therapists and education system including teachers and computer experts to the previous model.

We can finally associate this modelling to the actor-network theory. Michel Callon and Bruno Latour [2] developed it within the social sciences in order to explain for instance how innovations emerge. Therefore, the actors, as they are understood by this theory could be both human and non-human. When describing a network, it is important to underline the different relationships established between all the participants. Hélène Mialet [6] translated this theory within a new research she made on Stephen Hawkings public figure construction. She therefore shows how behind this unique person there is an entire network of actors (students, administrative assistants, nurses, a special computer, a special artificial voice, etc.) who are almost invisible. All of these will help us to better understand the position of the Student within our project and of the actors surrounding him.

\section{Methodology}

In order to better understand the previously described processes, as well as variations and similarities we could initially notice from one region to another and even inside a confined geographic area, we will focus our study on a practice analysis. That means we will focus on the analysis of those ensembles of patterned activities [5] we could identify within our work. Camis et al. [5] describe practices as particular ways in which the human being organises his or her activities: "[...] we define "practices" as the ensembles of patterned activities the "modes of working and doing," [...] by which human beings confront and structure the situated tasks with which they are engaged."

Subsequently, we will draw upon our own experience inside the project, as well as on documents exchanged between the different actors: meetings schedule; 
paper and electronic lists of students, tables of equipment and referees; Observation diaries of the experimenters.

We therefore combine and compare data gathered in this manner. Additional piece of information came from official websites or literature about the organisation of the educational system in France with a special point of view on inclusion for young people with disabilities.

\section{$7 \quad$ Work and Results}

Almost ten months after the beginning of the project, approximately 300 students were identified within the four regions. There are big variations, from 25 to 103 students per geographical area.

Students present different levels of implication: from the ones already using the device, to the ones who might be interested in doing this. If the initial inclusion criteria of students were strict, during the process of deployment they became more lax. For instance, besides the three categories of impairments addressed in the beginning, new requests were added for upper-limb motor impairments and other kinds of learning disabilities. Concerning the level of education, new categories were also included, for instance the lifelong learning training. Criteria concerning the level of computer expertise gradually opened themselves too.

A modification of the initial schedule was also observed. For instance, the first and the third phase sometimes mixed themselves. We can also have the case in which the third phase was not really implemented because of the length of the two previous ones, and also because there were school representatives who become direct referees for the student testing the device. The different participation of child-care actors and teachers influence the manner in which the technical object is used. The results are still processed, but the pedagogical part is not very visible for the moment. Moreover, if we look at the important number of meetings already organised during a short period of time (less than 6 month), we can say that the project, as well as the technical solution need to be introduced to their users. They need to learn how to use it, how to get used to it in order to deliver it around them, to the students "recruited" for the project.

The first observations of figure 1 show that even if we can identify a same category of actors, their actions are not the same through the different regions or academies. Each actor has its own demands and needs and this influences the general organisation of the experiment. In the same time, two big categories of actors accompany the young people within their learning process. They meet and interact in various ways, often they know each other, but sometimes they meet for the first time due to the need to work together in order to open the access of the students to the assistive technology. On the one hand, we can find the child-care professionals, on the other the public education representatives.

Child-care professionals are in general working in specialised centres, but there are also those belonging to private practices. An important number of centres appears in those regions were the number of participants students are higher. These actors accept very often to install the software on students computers, 
Table 1. Comparing the four regions

\begin{tabular}{lrrrr}
\hline & Region $\mathbf{1}$ & Region 2 & Region 3 & Region 4 \\
\hline Number of academies & 3 & 1 & 1 & 1 \\
\hline Child-care centres & 11 & 4 & 9 & 3 \\
\hline Public Education support & medium & medium & high & high \\
\hline Number of presentation & 14 & 1 & 9 & 3 \\
meetings with referees & $2-10$ ref. & 25 ref. & $3-15$ ref. & 5-20 ref. \\
\hline Number of training & 11 & 2 & 6 & 6 \\
meetings with referees & 3-10 ref. & 8-20 ref. & 5-15 ref. & $2-20$ ref. \\
\hline Number of presentation & Indirect & Together with & Together with & - \\
meetings with teaching & + email & the child-care & the child-care & \\
establishments & & centres & centres & \\
\hline University students & 23 & 9 & 1 & 19 \\
\hline Total students & 103 & 25 & 83 & 85 \\
\hline
\end{tabular}

but there are some exceptions too, when there are no IT professionals among them. An important part of them are speech therapists, orthoptists, child psychologists, or occupational therapists, but this last category often assists the technical learning process. As said before they meet the professionals from public education during the project. They can participate to the same meetings, or work with the same children.

Education professionals intervene less during the technical process. There are of course exceptions, especially when the child-care professionals do not assume this task. They are very diverse too, from inspectors to specialised teachers. The IT professionals often have an "expert" eye on the device, comparing it to similar solutions. Moreover, teachers and school officials participate to the identification of those young people who might be interested by the device. As mentioned before, their teaching role is less visible, but their one relationship to new technologies is important in how the novelty is received when the student is encouraged (or not) to use it.

Families also appear in relation with this testing process. They have to give their consent for their young children participation, but in the same time, some of them ask for supplementary information or insist on this participation seen as a way of making easier the manner in which they assist their children.

\section{Conclusion and Planned Activities}

Our paper underlines the main steps of our fieldwork, as well as its similarities and differences from the initial protocol. Is also calls attention on the fact that unexpected transformations can have an important impact on the resources and time allocated for the deployment. 
Some other actors than students look to be important during the technology learning and inclusion within school processes, even if their figure will not initially appear. We could therefore identify the role of some professionals like the occupational therapists within the health-care centres or the computer technicians within the EyeSchool system-installing phase.

This presentation emphasises how the experiment might not be neutral. Sometimes it can influence the general environment of the participating actors. By testing a technical tool, it might also arise additional questions, as in our case, questions about pedagogical practices or organisational ones. For instance, we can easily include in this last category those questions about providing computers for young people with disabilities who do not have one.

Finally, a more detailed phase of evaluation is planned to take place at the end of the school year. This is different from the testing process we have just described, as it looks at the answers the concerned actors give and less at their configuration and past history. A quantitative research was therefore designed. It uses as main tools a questionnaire for students, their families and the professionals that work with them, as well as the analysis of logs we could received after the period of test.

\section{References}

1. Ando, B., Baglio, S., La Malfa, S., Marletta, V.: Innovative Smart Sensing Solutions for the Visually Impaired. In: Pereira, J. (ed.) Handbook of Research on Personal Autonomy Technologies and Disability Informatics, ch. 5, pp. 60-74 (2010)

2. Callon, M., Latour, B.: Unscrewing the Big Leviathans. How Do Actors Macrostructure Reality. In: Knorr, K., Cicourel, A. (eds.) Advances in Social Theory and Methodology: Toward an Integration of Micro and Macro Sociologies, London, Routledge (1981)

3. Draffan, E.A., Evans, D.G., Blenkhorn, P.: Use of assistive technology by students with dyslexia in post-secondary education. In Disability and Rehabilitation: Assistive Technology 2(2), 105-116 (2007)

4. Lopez-Krahe, J.: Introduction to Assistive Technology for the Blind. The European Journal for the Informatics Professional 8(2), 4-9 (2007)

5. Camis, C., Gross, N., Lamont, M.: Social Knowledge in the Making. University of Chicago Press (2011)

6. Mialet, H.: Hawking incorporated: Stephen Hawking and the Anthropology of the Knowing Subject. The University of Chicago Press (2012)

7. Rekkedal, A.M.: Assistive Hearing Technologies Among Students With Hearing Impairment: Factors That Promote Satisfaction. Journal of Deaf Studies and Deaf Education 17(4), 499-517 (2012) 